\title{
Atrial Arrythmia in Atrial Septal Defect Patient
}

\author{
Indah Paranita, Lucia Kris Dinarti, Bambang Irawan \\ Department of Cardiology and Vascular Medicine, Faculty of Medicine, Universitas Gadjah Mada - \\ Dr. Sardjito Hospital, Yogyakarta, Indonesia \\ * correspondence: dr.alfarah@gmail.com
}

\begin{abstract}
Background: Atrial fibrillation (AF) and atrial flutter are the most common cardiac arrhythmias associated with atrial septal defects (ASD) in adult patients. The incidence could be as high as $52 \%$ in patients ages 60 years or more. Patient with congenital heart disease who developed atrial arrhythmias had a $>50 \%$ increased stroke risk. Nevertheless, studies regarding the pathophysiological mechanism underlying the high incidence of atrial fibrillation in adult patients with ASD remain relatively few.

Case presentation: A female 46 years referred to Dr. Sardjito Hospital with chest discomfort and palpitation. Physical examination revealed holosystolic murmur $3 / 6$ in lower sternal border and wide fixed split S2. Chest X Ray shown cardiomegaly with pulmonal artery is prominent. The ECG revealed atrial flutter, incomplete RBBB, RAD and RVH. Transthoracal echocardiography shown ASD with left to right shunt with diameter 1.2 -1.8 cm, LA, RA and RV dilatation, with normal systolic function. From right heart catetherization, the result is ASD with high flow low resistance, with pulmonary hypertension (mPAP $44 \mathrm{mmHg}$ ).

Discussion: The consequences of left to right shunt across an ASD is RV volume overload and pulmonary overcirculation. Atrial arrhytmia are a common result of long standing right side heart volume and pressure overload. Changes in atrial refractoriness, ionic currents and atrial conduction properties (electrical remodeling), along with tissue remodeling due to atrial fibrosis (structural remodeling) generate a favorable substrate for the initiation and domestication of AF. Prevention of atrial arrhythmias is one of the reason for repairing ASD in young symptomatic patient.

Conclusion: ASD is frequently associated with atrial arrhythmias and their incidence increase steeply with age. For the patient, the recommendation is surgical closure to improves exercise capacity, to prevents right to left shunt, and to eliminates heart failure. The idea of combining ASD closure and arrhythmia intervention is another approach to be considered.
\end{abstract}

